\title{
Assessment of facial nerve injury using Deep Subfascial approach to Temporo-mandibular joint
}

\author{
Ankit Kumar ${ }^{1}$, Sourav Kumar², Musaab Khan ${ }^{3}$, Dipti Nayak', Ashish Sharma ${ }^{5}$, \\ Siddhi Tripathi ${ }^{6}$ \\ ${ }^{1}$ Reader, Department of Oral and Maxillofacial Surgery, Kothiwal Dental College and Research Centre, Moradabad, UP, \\ ${ }^{2,3}$ Resident, Department of Oral and Maxillofacial Surgery, Kothiwal Dental College and Research Centre, Moradabad, \\ UP, ${ }^{4}$ Resident, Department of Prosthodontics Crown and Bridge, Kothiwal Dental College and Research Centre, \\ Moradabad, UP, ${ }^{5}$ Professor and Head, Department of Oral and Maxillofacial Surgery, ITS Dental College, Greater \\ Noida, UP, ${ }^{6}$ Professor, Department of Prosthodontics, ITS Dental College,Murad Nagar, Ghaziabad, UP
}

Background: Surgical access to the temporomandibular joint (TMJ) and zygomatic arch is a challenge even to the experienced maxillofacial surgeon. The conventional subfascial approach to these structures carries the potential risk of transient paralysis of the frontalis and orbicularis oculi muscles. The deep subfascial approach provides an additional layer of protection (the deep layer of the temporalis fascia and the superficial temporal fat pad) to the temporal and zygomatic branches of the facial nerve and thus, is the safest method to avoid facial nerve injury. Aims and Objective: To assess facial nerve injury (FN) following TMJ surgery using deep Subfascial approach and measuring it on House and Brackman facial nerve grading system (HBFNGS). Materials and Methods: A randomized study was performed from August 2013 to March 2017 on 24 patients with unilateral and bilateral TMJ ankylosis. All patients were evaluated objectively for facial nerve injury using with house and brackmann facial nerve injury grading system post-operatively and subjectively inthe various time periods, i.e. 24 hours, 1 week, 1 month, 3 months and 6 months. Results: Brackmann facial nerve grading system at 24 hours post operatively- in the deep subfascial approach group, $91.7 \%$ of patients (23 cases) had Grade 1 injury and $8.3 \%$ (1 case) had Grade 3 injury. The condition improved with time with full recovery of facial nerve injury (FN) at all surgical sites at 6 months. Conclusion: The deep subfascial approach has a distinct advantage over the conventional approaches when dissecting the temporal region and is the safest method to avoid injury to facial nerve injury (FN).
Website:

http://nepjol.info/index.php/AJMS DOI: 10.3126/ajms.v12i1.30784

E-ISSN: 2091-0576

P-ISSN: 2467-9100

Copyright (c) 2021 Asian Journal of Medical Sciences

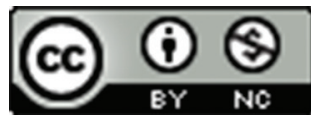

This work is licensed under a Creative Commons Attribution-NonCommercial 4.0 International License.

Key words: Temporomandibular Joint ankylosis; Deep subfascial; Nerve injury;

Assessment of facial nerve

\section{INTRODUCTION}

The temporomandibular joint (TMJ) is a commonly affected joint due to various ailments like ankylosis of the joint, condylar fractures, internal derangements, TMJ pathologies, zygomatico maxillary complex (ZMC) fractures, etc. which certainly require exposure of the joint and its nearby structures. However, there is a major limitation in the access to the joint, i.e. the facial nerve and its branches. While performing the surgical procedures on the TMJ the temporal branch is among the most endangered of the facial nerve branches to injury Contemporary literature reveals the incidence of facial nerve paresis which was found in $1.5 \%$ to $32 \%$ of patient. ${ }^{1,2}$ Modern day publications describe the three approaches for TMJ exposure: Dissection that is superficial to the superficial temporal fascia, ${ }^{3}$ exploitation of the surgical plane between the 2 layers of the temporalis fascia (subfascial) ${ }^{4}$ and an approach deep to both layers of the temporalis fascia (deep subfascial). ${ }^{5,67}$ This nerve lies in a condensation of the 
superficial fascia. ${ }^{11}$ Moreover, depletion in the facial nerve function impedes psychic expression, functional deficiency, and can lead to an esthetic deformity which might lead to overwhelming loss of quality of life. ${ }^{8,9}$

Recently, the deep subfascial approach has been advocated by many researchers stating that it is the safest approach in terms of preserving the integrity of the temporal branch of the facial nerve compared with the conventional approaches to the TMJ, because this nerve lies in a condensation of the superficial fascia. ${ }^{10-13}$ The present study aimed to assess facial nerve (FN) injury following TMJ surgery using deep subfascial approach and measuring it on House and Brackman facial nerve grading.$^{14,15}$

\section{Aims and objectives}

To assess FN injury following TMJ surgery using deep subfascial approach and measuring it on House and Brackman facial nerve grading system (HBFNGS).

\section{MATERIALS AND METHODS}

To concentrate on the study aim, the researchers outlined and executed a randomized controlled clinical trial which was carried out in the Department of Oral and Maxillofacial Surgery. The trial was sanctioned by the institutional review board and local ethics committee. The study followed the benchmarks as set by Helsinki. All patients without any systemic complications, who strictly satisfied the incorporating guidelines, were included. The study population was composed of all patients presenting for evaluation and management of unilateral and bilateral TMJ ankylosis from August 2013 to March 2017.To be included in the study sample, patients had tofulfil the following inclusion criteria: An established diagnosis of unilateral and bilateral TMJ ankylosis as proven by clinical and radiological diagnosis(Figure 1 and 2). Patients were excluded as study subjects if they were American Society of Anesthesiologist physical status classification system (ASA) III and IV compromised patients, had previous or current neurological disease that may adversely affect facial nerve function secondary to the previous or present neurological illness, patients who did not provide written informed consent, and patients who were not willing to attend follow-up appointments.

The diagnostic work-up was done for all patients; it included clinical examination and radiographic presentation. Standard lab investigations were done for all participants. Written informed consent was taken from all the subjects. Regardless of age and sex, the randomization of the patients was done using a slot method. In order to control the bias in the study, a single operator performed all the

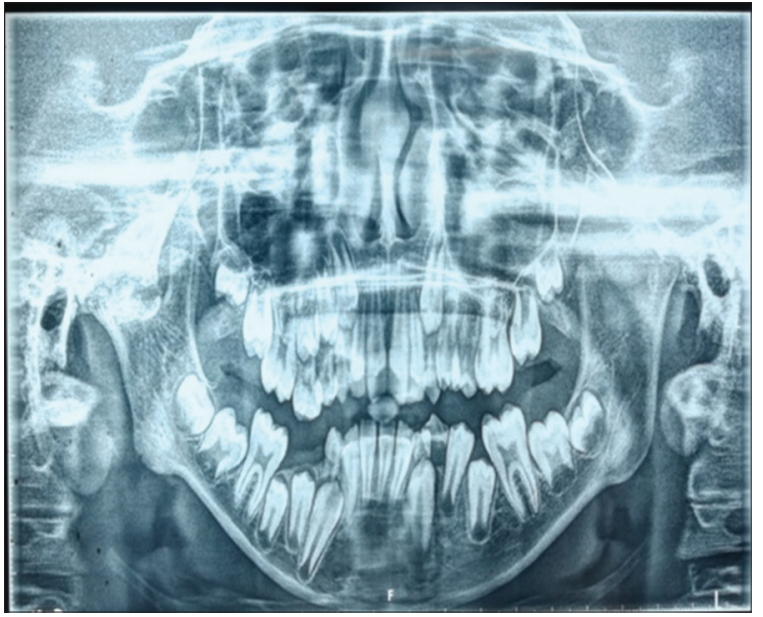

Figure 1: Showing right (unilaetral) TMJ anklosis

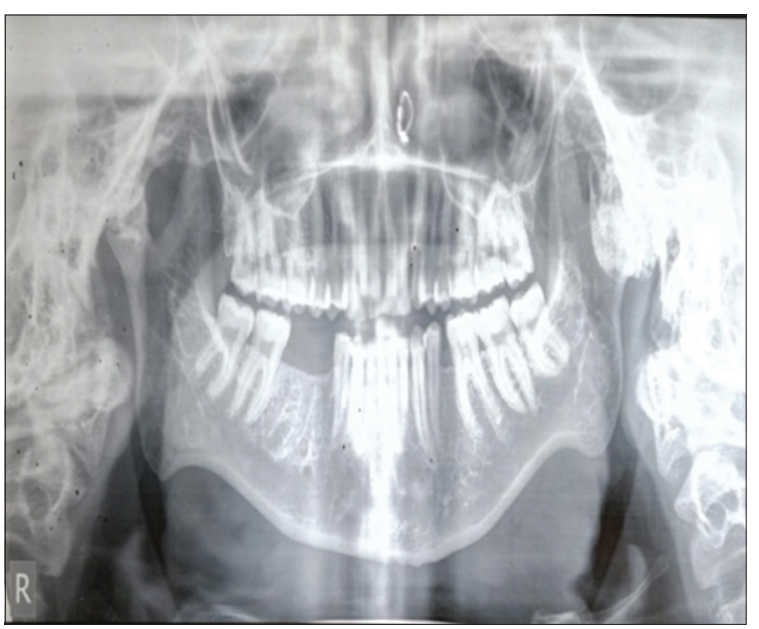

Figure 2: Showing bilateral TMJ ankylosis

surgeries under general anaesthesia along with standard aseptic provisions and protocol.

\section{Technique}

Skin preauricular incision extending to the temporal region, curving backwards and upwards posterior of the main branches of the temporal vessels was performed. The temporal component of the skin incision was made $45^{\circ}$ to the zygomatic arch, from the superior auriculocutaneous junction. The incision was carried through the subcutaneous tissue, the superficial temporalis fascia, and the areolar fat tissue. Blunt dissection is carried out downwards, to a point $2 \mathrm{~cm}$ above the malar arch where the deeptemporalis fascia splits into 2 layers containing fat tissue. Modification of the surgical technique places the incision of the upper and lower layer of the deeptemporalis fascia, completely through the fat tissue exposing the fibers of the temporal muscle and producing a new subfascial layer (under the deep temporal fascia) (Figure 3). Once inside this pocket, the periosteum of the malar arch can be safely incised and turned forward. The pocket can be developed anteriorly, allowing a safe and 
comfortable surgical approach to the articular capsule. This composite fasciocutaneous temporal flap includes: skin, subcutaneous tissue, superficial temporalis fascia, loose areolar tissue, superficial layer of deep temporalis fascia, temporal fat pad, and the deep layer of deep temporalis fascia. With this method we produce an additional protective fascial layer for the facial nerve. The dissection proceeds with meticulous running on the muscle fibers to the malar arch and capsule of TMJ (Figure 4). Finally, the fascial layer can be repositioned and sutured, covering the temporal muscle.

The following variables have been evaluated:

1. Facial Nerve Function: The House-Brackmann reviewing framework was utilized to survey motor function of the facial nerve ${ }^{15,16,17}$ It is a clinical strategy for assessing the facial nerve damage that is very extensive and incorporates vital things, for example, the presence of the frontal, periorbital and peribuccal musculature, both very still and in movement. It was presented in 1983 for clinical use and was changed by Brackmannin 1985. On the proposal of the Facial Nerve Disorders Committee it was formally

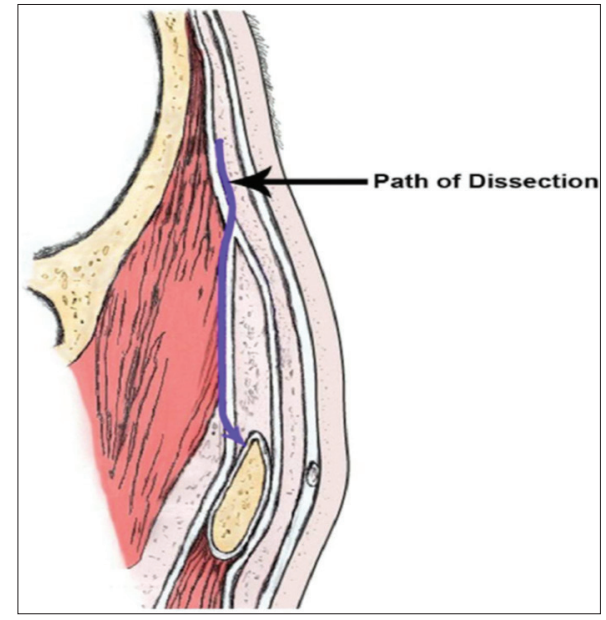

Figure 3: Dissection pathway for deep subfascial

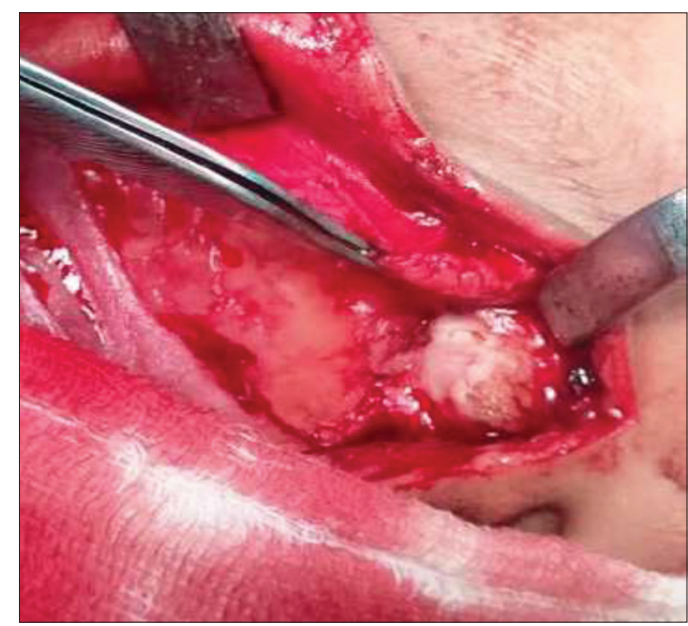

Figure 4: Surgical deep subfascial approach received as the general standard for detailing Facial Nerve work by the American Academy of Otolaryngology Head and Neck Surgery in 1984. It has accuracy of 93\% among the diverse evaluators. Appraisal capacity was done pre and postoperatively at $24 \mathrm{~h}, 1$ week, 1 month, 3 months, and a half year utilizing House-Brackmann Facial Nerve Grading System. The patients were analyzed in the accompanying positions: very still, raising the eyebrows, shutting the eyes with least exertion and with maximal endeavor, blowing the mouth.

All twenty four patients were treated for unilateral and bilateral TMJ ankylosis.

All patients were evaluated objectively for facial nerve function test before surgery (D0) and post-surgically after 24 hours (D1), 7days (D2), 30 days (D3), 90 days (D4), 180days (D5). Moreover, in order to determine the quality of life all the patients were followed post-surgically, at 1 month and 6 months. However, in order to control the bias, the Nerve Function Test and quality of life questionnaire was evaluated by another surgeon. Patients were re-evaluated for any clinical recurrence on all visits. Computed Tomography (CT scan) was advised if clinical examinations revealed any doubt.

\section{RESULTS}

The present study was conducted on 24 patients of unilateral TMJ ankylosis Assessment of facial nerve injury using deep subfascial approach to Temporo-mandibular joint

Analysis of facial nerve injury was done using House Brackmann System

The House Brackmann system was used to analyze the grades of facial nerve injury at various time periods explained:

Time periods, i.e. 24 hours, 1 week, 1 month, 3 months and 6 months, respectively. The results are

1) House-Brackmann facial nerve grading system at 24 hours post operatively-in the deep subfascial approach group, $91.7 \%$ of patients ( 22 cases) had Grade 1 injury and $8.3 \%$ ( 2 case) had Grade 3 injury (Table 1).

2) House-Brackmann facial nerve grading system at 1 -week post operatively in the deep subfascial approach, $100 \%$ of patients had grade 1 injury, i.e. the nerve function became normal in all the patients of deep subfascial group (Table 2).

3) House-Brackmann facial nerve grading system at 1 -month post operatively in the deep subfascial 
approach, $100 \%$ of patients continued to have grade 1 injury, i.e. the nerve function remained normal in all the patients (Table 3).

4) House- Brackmann facial nerve grading system at 3 months post operatively in the deep subfascial approach, $100 \%$ of patients (24 cases) continued to be in the grade 1 category i.e. normal functioning of the facial nerve remained for all patients (Table 4).

5) House-Brackmann facial nerve grading system at 6 month post operatively. In the deep \subfascial approach, $100 \%$ of patients (24 cases) remained in grade 1 facial nerve injury.

Complete recovery at all surgical sites proves the point that the deep subfascial approach is the safest among the preauricular approaches as far as facial nerve injury (FN) is concerned.

No sign of infection was observed in any patient in the follow-up appointments. Presence of Frey's syndrome defined as "perspiration of skin around the preauricular area while eating" was assessed on follow at 1 week, 1 month, 3 months, and 6 months postoperatively and was not evident in any of the patients.

In all the surgical sites, at 6 months follow-up, scar was imperceptible and esthetically acceptable.

\section{DISCUSSION}

The main purpose of this study was to evaluate the safety of a deep subfascial approach over on facial nerve injury following management of unilateral and bilateral TMJ ankylosis. Over the years, a number of surgical approaches to TMJ have been developed to attain the goal of successful removal of ankylotic mass, treating TMJ pathologies and condylar fracture.

In a study conducted by do Egito Vasconcelos BC et alon facial nerve function after surgical procedures for the treatment of temporomandibular pathology using House-Brackmann facial nerve grading system (HBFNGS) on 32 patients with 50 joints pathology using subfascial approach, they found that of 32 patients, $12.5 \%$ (8\% of the 50 approaches) revealed signs of facial nerve injury. There was a significant amount of post operative facial nerve injury in the patients who underwent surgery for TMJ ankylosis $(\mathrm{p}=0.014)$ and for gap arthroplasty patients $(p=0.014)$. The study reveals that at 24 hours, none of the patients showedtotal nerve paralysis or severe dysfunction, only a moderately severe dysfunction (50\%), or moderate dysfunction $(50 \%)$. The forehead area was the most commonly affected. However, at 3 months follow-up, all

\begin{tabular}{|c|c|c|}
\hline \multirow[t]{2}{*}{ Grade } & \multicolumn{2}{|c|}{ Deep subfascial approach } \\
\hline & $\mathbf{N}$ & $\%$ \\
\hline Grade 1 & 22 & 91.7 \\
\hline Grade 2 & 0 & 0 \\
\hline Grade 3 & 2 & 8.3 \\
\hline Grade 4 & 0 & 0 \\
\hline Grade 5 & 0 & 0 \\
\hline Grade 6 & 0 & 0 \\
\hline
\end{tabular}

\begin{tabular}{lcc}
$\begin{array}{l}\text { Table 2: Distribution of patients:House- } \\
\text { Brackmann facial nerve grading system at } \\
\text { 1-week post operatively }\end{array}$ \\
\cline { 2 - 3 } Grade & Deep subfascial approach \\
\cline { 2 - 3 } & $\mathbf{N}$ & $\%$ \\
\hline Grade 1 & 24 & 100 \\
Grade 2 & 0 & 0 \\
Grade 3 & 0 & 0 \\
Grade 4 & 0 & 0 \\
Grade 5 & 0 & 0 \\
Grade 6 & 0 & 0 \\
Total & 24 & 100 \\
\hline
\end{tabular}

\begin{tabular}{lcc} 
Table 3: Distribution of patients among:House- \\
Brackmann facial nerve grading system at \\
1-month post operatively \\
\hline Grade & \multicolumn{2}{c}{ Deep subfascial approach } \\
\cline { 2 - 3 } & $\mathbf{N}$ & $\%$ \\
\hline Grade 1 & 24 & 100 \\
Grade 2 & 0 & 0 \\
Grade 3 & 0 & 0 \\
Grade 4 & 0 & 0 \\
Grade 5 & 0 & 0 \\
Grade 6 & 0 & 0 \\
Total & 24 & 100 \\
\hline
\end{tabular}

\begin{tabular}{lcc} 
Table 4: Distribution of patients: House- \\
Brackmann facial nerve grading system at \\
3 month post operatively \\
\cline { 2 - 2 } Grade & Deep subfascial approach \\
\cline { 2 - 3 } & $\mathbf{N}$ & $\%$ \\
\hline Grade 1 & 24 & 100 \\
Grade 2 & 0 & 0 \\
Grade 3 & 0 & 0 \\
Grade 4 & 0 & 0 \\
Grade 5 & 0 & 0 \\
Grade 6 & 0 & 0 \\
Total & 24 & 100 \\
\hline
\end{tabular}

patients acquired normal facial nerve function. ${ }^{16}$ Our study showed similar results, however, there was no significant injury present at the six months time interval. This high frequency of nerve injury in our study during a subfascial approach, up to a period of 1 month, could have been 
either due to heavy retraction causing compression and/ or stretching of nerve fibre resulting in neuropraxia, or in a few cases it could have been due to inadvertent suture ligation of facial nerve fibers. Politi et al applied the deep subfascial approach to 21 patients and did not observe any temporary or permanent facial nerve function loss.

They reported that the facial nerve had been safely avoided, and the function of the auricular temporal nerve was also preserved. ${ }^{10}$ Similarly, Kenkere et al carried out a detailed study on 12 patients and made 15 surgical exposures to access the TMJ and zygomatic arch. They used a deep subfascial approach and found that no functional deficit was observed in either the temporal or zygomatic branches of the facial nerve as ascertained by clinical examination. ${ }^{12}$ Likewise, in our study, of 12 patients in whom a deep subfascial approach used, only one patient had grade 3 nerve injury; the remaining 11 showed grade 1 nerve injury at 24 hour post operatively. The low frequency of nerve injury in our study during deep subfascial approach. The evaluation was done in terms of facial nerve injury using House-Brackmann grading. The present study used the Face instrument to analyse patient perception regarding the approaches. The results of the study clearly indicate that no significant difference exists between the two approaches, in consideration of facial nerve injury during long term follow-up. ${ }^{9}$ Further studies with a larger number of cases and multicenter involvement can be done for a more definitive conclusion.

Although during 6-month follow up both the approaches show $100 \%$ recovery, still we found that a deep subfascial approach is a better approach during the initial follow-up of the patient in terms of facial nerve injury, since this technique provides an additional layer of protection (the deep layer of the temporalis fascia and the superficial temporal fat pad) to the temporal and zygomatic branches of the facial nerve. The plane of dissection is distinctly identifiable and reliable, and the technique is simple to use with basic knowledge of the anatomy of the region. This technique is indicated for any surgery of the TMJ, including ankylosis and zygomatic arch and especially for secondary surgeries in the temporal region and TMJ, as well as correction of post-traumatic deformity of the zygomatic arch and complex.

\section{CONCLUSION}

A deep subfascial approach has proven to be a safer surgical procedure with respect to facial nerve injury as compared to a routine subfascial approach, although long term follow-ups render the differences between the approaches insignificant. Nonetheless, a deep subfascial approach can be considered for both routine and complex TMJ ankylosis cases.

\section{REFERENCES}

1. Nellestam $P$ and Eriksson L. Preauricular approach to the temporomandibular joint: A postoperative follow-up on nerve function, hemorrhage and esthetics. Swed Dent J.1997;21(12):19-24.

doi.org/10.1016/j.joms.2006.06.280

2. Alsén B, Svensson B and Adell R. Facial nerve injuries in temporomandibular joint surgery. Tandlakartidningen. 1994;18:1157.

3. Gosain AK, Sewall SR and Yousif NJ. The temporal branch of the facial nerve: How reliably can we predict its path? Plast Reconstr Surg. 1997;99(5):1224-1233; discussion 1234-1236. https://doi.org/10.1097/00006534-199705000-00003

4. Al-Kayat A, Bramley $\mathrm{P}$ : A modified pre-auricular approach to thetemporomandibular joint and malar arch. $\mathrm{Br} \mathrm{J}$ Oral Surg. 1979; 17:91.

https://doi.org/10.1016/S0007-117X(79)80036-0

5. Rowe NL: Surgery of the temporomandibular joint. Proc R Soc Med.1972; 65:383.

https://doi.org/10.1177/003591577206500438

6. Toscano P. The temporofrontalfaciocutaneous flap in orbito malar zygomatic surgical approach, in Proceedings of the VIII Annual Meeting of the Italian Society of Maxillofacial Surgery, May 4-9, 1993, Rome, Italy. Bologna, Italy, Monduzz Editore, 1993.

7. Hochberg J, Kaufman $\mathrm{H}$ and Ardenghy M. Saving the frontal branch during a low front orbital approach.Aesthetic Plastic Surgery. 1995; 19: 161-163.

https://doi.org/10.1007/BF00450252

8. Satoh $\mathrm{Y}$, Kanzaki $\mathrm{J}$ and Yoshihara S. A comparison and conversion table of 'the House-Brackmann facial nerve grading system' and 'the Yanagihara grading system'. AurisNasus Larynx. 2000; 27:207-212.

https://doi.org/10.1016/S0385-8146(99)00049-8

9. Kahn JB1, Gliklich RE, Boyev KP, Stewart MG, Metson RB and McKenna MJ. Validation of a patient-graded instrument for facial nerve paralysis: the FaCE scale. Laryngoscope. 2001;111(3):387-398.

https://doi.org/10.1097/00005537-200103000-00005

10. Politi M, Toro C, Cian R, Costa F and Robiony M. The deep subfascial approach to the temporomandibular joint.J Oral Maxillofac Surg. 2004;62(9):1097-1102.

https://doi.org/10.1016/j.joms.2003.10.013

11. Candirli $\mathrm{C}$ and Celik S. Efficacy of deep subfascial approach to the temporomandibularjoint. J Craniofac Surg. 2012;23(2):e126-e129.

https://doi.org/10.1097/SCS.0b013e31824cdb5c

12. Kenkere D, Srinath KS and Reddy M. Deep subfascial approach to the temporal area. J Oral Maxillofac Surg. 2013;71(2):382-388. https://doi.org/10.1016/j.joms.2012.04.019

13. Malhotra V, Dayashankara Rao JK, Arya V, Sharma S, Kataria Y and Luthra P.Assessment of facial nerve injury with "House and Brackmann facial nerve grading system" in patients of temporomandibular joint ankylosis operate using deep subfascial approach. Natl J Maxillofac Surg. 2015;6(2):194-199. https://doi.org/10.4103/0975-5950.183876

14. House JW and Brackmann DE. Facial nerve grading system. 
Otolaryngol Head Neck Surg. 1985;93(2):146-147.

https://doi.org/10.1177/019459988509300202

15. House JW. Facial nerve grading systems. Laryngoscope. 1983;93(8):1056.

https://doi.org/10.1288/00005537-198308000-00016

16. Vasconcelos BC, Bessa-Nogueira RV and Da Silva LC. Prospectivestudy of facial nerve function after surgical procedures for the treatment of temporomandibular pathology.J Oral Maxillofac Surg. 2007;65(5):972-978.

https://doi.org/10.1016/j.joms.2006.06.280

17. Rattan V, Sharma VK, Rai SK and Malhi P. Quality of life in patients after temporomandibular joint ankylosis release. Int $\mathrm{J}$ Oral Maxillofac Surg. 2017;46(S1): 362-363.

https://doi.org/10.1016/j.ijom.2017.02.1220

\footnotetext{
Author's Contribution:

Work attributed to:

Kothiwal Dental College and Research Centre, Moradabad, UP

\section{Orcid ID:}

Dr. Ankit Kumar - (1) https://orcid.org/0000-0003-4153-8301

Dr. Sourav Kumar - (D) https://orcid.org/0000-0003-2140-5886

Dr. Musaab Khan - (D) https://orcid.org/0000-0001-8194-7682

Dr. Dipti Nayak - ic https://orcid.org/0000-0003-0068-4126

Dr. Ashish Sharma - (1) https://orcid.org/0000-0001-7172-0898

Dr. Siddhi Tripathi - (D) https://orcid.org/0000-0003-4000-4113
}

AK - Concept and design of the study; interpreted the results, prepared first draft of manuscript and critical revision of the manuscript; SK - Statistically analyzed and interpreted; reviewed the literature and manuscript preparation; MK - Design of the study, DN - statistically analyzed and interpreted, preparation of manuscript and revision of the manuscript; AS - Concept and ST - coordination of the overall study.

Source of funding: None, Conflict of Interest: None. 